IN HUMBOLDT'S SHADOW 



\title{
In Humboldt's Shadow
}

\author{
A TRAGIC HISTORY OF \\ GERMAN ETHNOLOGY
}

RN.

H. Glenn Penny 
English language copyright (C) 2021 by Princeton University Press

Originally published as Im Schatten Humboldts by H. Glenn Penny (C) Verlag C.H.Beck oHG, München 2019

Princeton University Press is committed to the protection of copyright and the intellectual property our authors entrust to us. Copyright promotes the progress and integrity of knowledge. Thank you for supporting free speech and the global exchange of ideas by purchasing an authorized edition of this book. If you wish to reproduce or distribute any part of it in any form, please obtain permission.

Requests for permission to reproduce material from this work should be sent to permissions@press.princeton.edu

Published by Princeton University Press

41 William Street, Princeton, New Jersey 08540

6 Oxford Street, Woodstock, Oxfordshire OX20 1TR

press.princeton.edu

All Rights Reserved

Library of Congress Cataloging-in-Publication Data

Names: Penny, H. Glenn, author.

Title: In Humboldt's shadow : a tragic history of German ethnology / H. Glenn Penny.

Other titles: Im Schatten Humboldts. English

Description: Princeton, New Jersey : Princeton University Press, 2021. |

Includes bibliographical references and index.

Identifiers: LCCN 2020049825 (print) | LCCN 2020049826 (ebook) |

ISBN 9780691211145 (hardback) | ISBN 9780691216454 (ebook)

Subjects: LCSH: Ethnology-Germany-History. | Ethnologists-Germany-History. |

Ethnological museums and collections-Germany-History. | Humboldt, Alexander von, 1769-1859-Influence.

Classification: LCC GN308.3.G3 $\mathrm{P}_{4} 6132021$ (print) | LCC GN308.3.G3 (ebook) |

DDC $305.800943-\mathrm{dc} 23$

LC record available at https://lccn.loc.gov/2020049825

LC ebook record available at https://lccn.loc.gov/2020049826

British Library Cataloging-in-Publication Data is available

Editorial: Fred Appel and James Collier

Production Editorial: Kathleen Cioffi

Jacket Design: Amanda Weiss

Production: Erin Suydam

Publicity: Kate Hensley and Kathryn Stevens

Copyeditor: Anne Cherry

Jacket image: iStock

This book has been composed in Miller

Printed on acid-free paper. $\infty$

Printed in the United States of America

$109876 \begin{array}{llllll} & 8 & 4 & 32 & 1\end{array}$ 